\title{
Total (food and supplement) n-3 PUFA intake is associated with lower Coronary Heart Disease mortality, independently of fish intake
}

\author{
M.A.H. Lentjes ${ }^{1}$, R.H. Keogh ${ }^{2}$, A.A. Welch ${ }^{3}$, A.A. Mulligan ${ }^{1}$, R.N. Luben ${ }^{1}$ and K.T. Khaw ${ }^{4}$ \\ ${ }^{1}$ University of Cambridge, Department of Public Health \& Primary Care, Cambridge CB1 8RN, ${ }^{2}$ London School of \\ Hygiene and Tropical Medicine, London, ${ }^{3}$ University of East Anglia, Department of Population Health \& Primary \\ care, Norwich Medical School, Norwich NR4 7TJ and ${ }^{4}$ University of Cambridge, Clinical Gerontology Unit, \\ Cambridge CB2 $2 Q Q$
}

Fish contains essential polyunsaturated fatty acids (n-3 PUFA) which increase n-3 PUFA concentrations in the cardiac membrane and influence cardio electrophysiology, which might have antiarrhythmic effects and so lower risk of fatal $\mathrm{CHD}{ }^{(1,2)} \mathrm{Clinical}$ trials of n-3 PUFA supplements conducted in high risk populations show no significant benefit, ${ }^{(3)}$ results from observational studies on fish intake show heterogeneous results. ${ }^{(4)}$ N-3 PUFA containing supplements, mainly cod liver oil, are widely used in the UK and by $24 \%$ in the Norfolk-based European Prospective Investigation into Cancer (EPIC-Norfolk). ${ }^{(5,6)}$ We studied the association between n-3 PUFA Total Nutrient Intake (TNI, i.e. intake from foods and supplements), n-3 PUFA supplement use and fatal CHD in a general population-based cohort.

EPIC-Norfolk recruited men and women, between 39-79 y $(\mathrm{N}=25,639)$ between 1993-1997. Anthropometry was measured. Participants completed a 7-day diet diary, from which n-3 PUFA TNI, energy intake and disaggregated food consumption were determined. Participants were classified into three groups: non-supplement users (NSU), supplement users without n-3 PUFA supplements (SU-n3) and supplement users with n-3 PUFA supplements (SU+n3). General questionnaires ascertained social class, education, smoking, physical activity, alcohol consumption and prevalent diseases. Analyses were based on $\mathrm{n}=22,137$ with complete data. After a median follow-up of 18 years, 1393 participants died from CHD (ICD 410-414/I20-25). Cox proportional hazards regression was used to analyse differences between supplement groups as well as quintiles (Q5 $v$ Q1) of TNI intake.

$\mathrm{SU}+\mathrm{n} 3$ (compared to NSU) were more likely to be women, >60 years, and to be non-smokers and alcohol consumers. They reported fewer higher educational qualifications and less physical activity. SU $+\mathrm{n} 3$ and SU-n3 had lower self-reported history of myocardial infarction, diabetes or stroke. Differences in median (Med) n-3 sourced intake are shown in the top half of the table. SU + n3 did not have lower risk of fatal CHD; however higher n-3 PUFA intake was associated with a $22 \%$ lower risk of fatal CHD, after adjusting for fish consumption, indicating that other sources than fish are associated with fatal CHD.

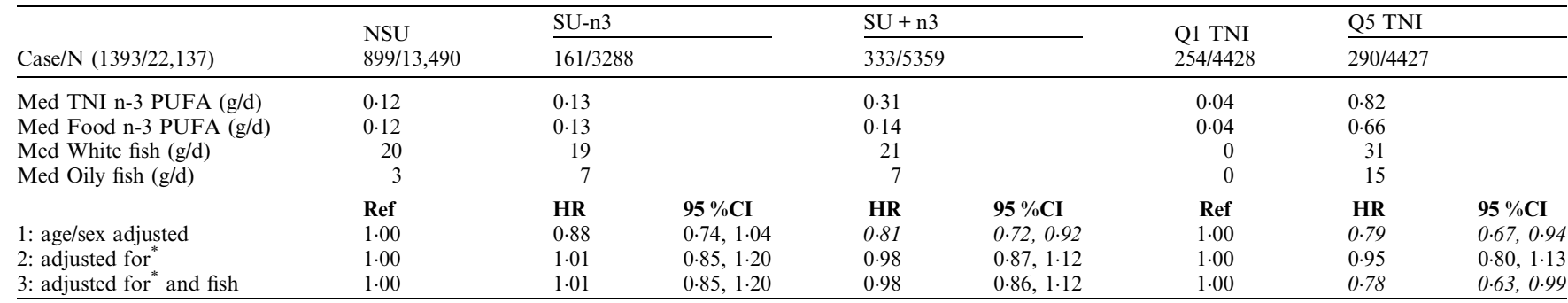

${ }^{*}$ sex, age, smoking, body mass index, alcohol, social class, education, season, physical activity, energy intake, fruit, vegetables, red meat, processed meat, white meat, prevalent diabetes/stroke/myocardial infarction.

Non-fish n-3 PUFA was negatively associated with fatal CHD. The negative confounding observed from fish might be explained by preparation methods $^{(7)}$ or UK dietary patterns (fish ' $n$ chips); alternatively, contamination of fish with methylmercury might play a role. ${ }^{(8)}$

1. London B et al. (2007) Circulation 116, e320-e335; 2.

2. Mozaffarian D et al. (2006) JAMA 296, 1885-1899; 3.

3. Chowdhury R et al. (2014) Ann. Intern. Med. 160, 398-406; 4.

4. Zheng J et al. (2012) Public Health Nutr. 15, 725-737; 5.

5. Lentjes MAH et al. (2014) Nutrients 6, 4320-37; 6.

6. Lentjes MAH et al. (2014) J. Hum. Nutr. Diet.; 7.

7. Mozaffarian D et al. (2003) Circulation 107, 1372-1377; 8.

8. Stern AH (2007) Environ. Health 6, 31. 